\title{
Association between personality factors and consulting specialty of practice of doctors at an academic hospital in Bloemfontein,
}

\section{South Africa}

R van Aswegen, ${ }^{1}$ Undergraduate medical student; A Ravgee, ${ }^{1}$ Undergraduate medical student; G Connellan, ${ }^{1}$ Undergraduate medical student; C Strydom, ${ }^{1}$ Undergraduate medical student; J T Kuzhivelil, ${ }^{1}$ Undergraduate medical student; G Joubert, ${ }^{2}$ BA, MSc;

W J Steinberg, ${ }^{3}$ MB BCh, DTM\&H, DPH, Dipl Obst (SA), MFamMed, FCFP (SA)

${ }^{1}$ School of Medicine, Faculty of Health Sciences, University of the Free State, Bloemfontein, South Africa

${ }^{2}$ Department of Biostatistics, Faculty of Health Sciences, University of the Free State, Bloemfontein, South Africa

${ }^{3}$ Department of Family Medicine, Faculty of Health Sciences, University of the Free State, Bloemfontein, South Africa

Corresponding author: W J Steinberg (steinbergwj@ufs.ac.za)

Background. Studies found an association between personality types and field of specialty. The current study could assist aspiring specialists in deciding which specialty they are best suited for by comparing their own personalities with the results.

Objectives. To explore the personality characteristics of doctors in three consulting and four surgical specialties at an academic hospital in Bloemfontein, South Africa.

Methods. In this analytical cross-sectional study, questionnaires, including the Zuckerman-Kuhlman Personality Questionnaire, were handed out. Overall, 58 consultants and senior registrars from the departments of Family Medicine, Paediatrics and Internal Medicine (response rate $71.6 \%$ ) and 70 consultants and senior registrars from surgical specialties (response rate 60.3\%) participated.

Results. Family medicine had the lowest median score for impulsive sensation seeking (21.1\%) and aggression-hostility (11.8\%), and highest for parties and friends (33.3\%). Paediatrics scored highest for neuroticism-anxiety (44.7\%) and aggression-hostility (23.5\%). Internal medicine scored highest for sociability (25.0\%) and isolation intolerance (37.8\%), and lowest for neuroticism-anxiety (36.8\%) and activity (47.1\%). Overall, the consulting group scored lower than the surgical group for impulsive sensation seeking, aggression-hostility, sociability and activity, and higher for neuroticism-anxiety. Conclusion. The study identified personality types of some specialties, and revealed differences between characteristics of local specialists compared with findings from studies elsewhere.

Afr J Health Professions Educ 2018;10(2):79-84. DOI:10.7196/AJHPE.2018.v10i2.997

The concept of personality as a measurable trait is hindered by the lack of consensus in the field of psychology on the appropriate model of personality. Personality psychologists have attempted to attach biological functions to traits and the diversity of traits, and proposed numerous models..$^{[1,2]}$ The alternative five factorial model of personality claims that human personality can be explained by five broad factors ${ }^{[3]}$ that have a strong biologicalevolutionary basis. ${ }^{[4]}$ These five factors are: ${ }^{[3]}$

- Impulsive sensation seeking: 'The tendency to act quickly on impulse without planning, often in response to a need for thrills and excitement, change and novelty.'

- Neuroticism-anxiety: 'The tendency to be tense and worry, overly sensitive to criticism, easily upset, and obsessively indecisive.'

- Aggression-hostility: 'The tendency to express verbal aggression and show rudeness, thoughtlessness, vengefulness, spitefulness, a quick temper and impatient behaviour.'

- Sociability: 'Tendencies to interact with others, enjoyment in being with others, and intolerance for social isolation.'

- Activity: 'The tendency to be active, to prefer challenging work, and being impatient or restless when there is nothing to do.'
Numerous studies have attempted to classify under- and postgraduate medical students and specialists into personality categories. ${ }^{[4-10]}$ A study ${ }^{[5]}$ published in 2004 used the Cloninger Inventory to identify the influence of temperament on medical students' choice of specialty. They distributed the questionnaire to 682 medical students with a choice of preference from procedure-orientated specialties (surgery, obstetrics and gynaecology) and primary care specialties (family medicine, internal medicine, paediatrics). Procedure-orientated specialties had higher novelty-seeking scores and scored high on co-operativeness and self-directedness. Those interested in emergency medicine scored high on novelty-seeking and low on harmavoidance scales, similar to surgeons, but higher in reward dependence than surgeons. Students choosing primary care specialties, especially paediatrics, had the highest reward-dependence scores. Those choosing internal medicine had higher harm-avoidance scores than those choosing surgery and emergency medicine.

Hojat and Zuckerman ${ }^{[3]}$ tested the personalities of 1076 medical students at Jefferson Medical College, Philadelphia, USA, between 2002 and 2006, according to specialty interest. The study used the Zuckerman-Kuhlman Personality Questionnaire (ZKPQ) measuring the five personality factors 
mentioned above. It showed that those interested in surgical specialties had higher than average scores for impulsive sensation-seeking and aggressionhostility factors, and lower scores in the neuroticism-anxiety scale. Those interested in obstetrics and gynaecology were more often females and scored highest in neuroticism-anxiety. Those interested in hospital care scored lower on the sociability scale, while those interested in emergency medicine and surgery had high activity scores, especially compared with psychiatry students.

Many doctors and medical students are faced with the dilemma of whether to specialise. To specialise in a field of medicine is often a lifelong commitment - one that requires careful consideration. We believe that perceptions of the type of personalities of individuals in a specific specialty may influence the choice of specialty, and many of these perceptions may be based on stereotypes. The current study could assist aspiring specialists in deciding which specialty they are best suited for by comparing their own personalities with the results.

\section{Objectives}

The aim of the study was to explore any association between the personality factors and consulting specialty of practice of doctors at an academic hospital in South Africa (SA) in 2014. Furthermore, to provide a broader context, the overall results for consulting and surgical groups are provided.

\section{Methods \\ Design and setting}

This was an analytical cross-sectional study conducted over 4 months, from August to November 2014, at an academic hospital in Bloemfontein, SA.

\section{Population and sampling strategy}

The target population consisted of 197 doctors working as senior registrars or consultants at 7 specialty departments at the academic hospital. The specialties were assigned to the 'consulting group' (family medicine, internal medicine and paediatrics) or the 'surgical group' (anaesthesiology, obstetrics and gynaecology, orthopaedic surgery and surgery). This article focuses mainly on the consulting group, which consisted of 81 doctors.

\section{Inclusion criteria}

Senior registrars (in their third or fourth year of the MMed degree) and specialist consultants, of all ages, who practised at the academic hospital were included. There were no exclusion criteria.

\section{Method of sample selection}

Convenience sampling of all consultants and senior registrars present at departmental meetings was performed, unless they declined. Completed questionnaires were retrieved at the meeting or from the departmental secretary if these were not completed at the meeting.

\section{Data collection}

The psychometric tool used in this study was the ZKPQ. ${ }^{[11]}$ It measures five factors of personality: (i) impulsive sensation seeking; (ii) neuroticismanxiety; (iii) aggression-hostility; (iv) sociability; and ( $v$ ) activity. Impulsive sensation seeking is subdivided into impulsivity and sensation seeking. Sociability is subdivided into parties and friends, and isolation intolerance, and activity is subdivided into work effort and general activity.
The ZKPQ, a valid self-report measure, ${ }^{[12]}$ consists of 99 items in true/ false format. In addition to the ZKPQ, a section was added to the study questionnaire to capture demographic data such as age, gender and years in field of specialty. The questionnaire was available only in English.

\section{Pilot study}

Eight medical interns training at the academic hospital were included in the pilot study. A few minor adjustments were made to the questionnaire. The data collected were not included in the study.

\section{Data analysis}

Data were analysed by the Department of Biostatistics, Faculty of Health Sciences, University of the Free State (UFS), Bloemfontein. Results are presented as median percentages of the maximum result for each specific scale because of skew data distributions. For each scale, the score obtained was expressed as a percentage of the maximum total score applicable for that scale. If $>20 \%$ of the questions for a specific scale were not answered, the participant's result for that scale was excluded from the results.

The ZKPQ includes a sixth scale, i.e. infrequency, which identifies participants who provide invalid test results by selecting responses that are unlikely to be true ${ }^{[3]}$ A comparison was made between results that excluded questionnaires with scores $>30 \%$ on the infrequency scale and results that did not exclude questionnaires with scores $>30 \%$. A few differences were noted, but these were not significant enough to warrant the use of the results that excluded questionnaires.

Statistical analysis was performed using the non-parametric MannWhitney test, and $95 \%$ confidence intervals (CIs) for the median differences were calculated. Values $p<0.05$ were considered statistically significant.

\section{Ethical approval}

The protocol was approved by the Ethics Committee of the Faculty of Health Sciences, UFS (ref. no. STUD no 28/2014). Permission to conduct the study was obtained from the heads of the academic departments, as well as from the head of clinical services at the academic hospital. Permission to use the ZKPQ in the study was given via email by Prof. Marvin Zuckerman (co-creator), along with the manual that was used to score each individual's results.

\section{Results}

The response rates from each consulting specialty and the overall consulting and surgical groups are shown in Table 1.

Family medicine was the consulting specialty with the highest median age (54 years) and duration of practice (14.5 years) (Table 2). All consulting specialties had higher medians than the surgical group (age: $p<0.01$;

\begin{tabular}{llll} 
Table 1. Sample and population sizes & & \\
\hline & $\begin{array}{l}\text { Sample } \\
\text { size, } \boldsymbol{n}\end{array}$ & $\begin{array}{l}\text { Population } \\
\text { size, } \boldsymbol{n}\end{array}$ & $\begin{array}{l}\text { Response } \\
\text { rate, } \%\end{array}$ \\
Specialty & 13 & 16 & 81.3 \\
Family medicine & 25 & 37 & 67.6 \\
Internal medicine & 20 & 28 & 71.4 \\
Paediatrics & 58 & 81 & 71.6 \\
Consulting group, all & 70 & 116 & 60.3 \\
Surgical group, all & & &
\end{tabular}


95\% CI 2 - 10 years; duration of practice: $p<0.01 ; 95 \%$ CI 1 - 6 years). All consulting specialties had a larger percentage of females than the surgical specialties $(p=0.03)$.

\section{Impulsive sensation seeking}

Family medicine had the lowest median score (21.1\%) for impulsive sensation seeking (Fig. 1). All three consulting specialties had medians of $36.4 \%$ for the subscale sensation seeking. Family medicine and paediatrics both had medians of 0 for the subscale impulsivity. All three consulting specialties had lower medians than the overall surgical group for impulsive sensation seeking ( $p<0.01 ; 95 \%$ CI $-25.8 \%$; $-5.3 \%)$ and the subscale sensation seeking ( $p=0.01 ; 95 \%$ CI $-27.3 \% ;-3.6 \%$ ). The subscale sensation seeking scored higher than impulsivity across consulting and surgical groups.

\section{Neuroticism-anxiety}

Paediatrics scored the highest $(44.7 \%)$ and internal medicine the lowest (36.8\%) for neuroticism-anxiety. The consulting group scored higher than the surgical group ( $p=0.06 ; 95 \%$ CI $0 ; 15.8 \%$ ) (Fig. 2 ).

\section{Aggression-hostility}

Paediatrics scored the highest (23.5\%) and family medicine the lowest (11.8\%) for aggression-hostility (Fig. 3). There was a strong distinction between the two major groups, with the consulting group scoring much lower than the surgical group ( $p<0.01 ; 95 \%$ CI $11.8 \% ; 23.5 \%)$.

\section{Sociability}

Internal medicine had the highest score for sociability (25.0\%) and the subscale isolation intolerance (37.8\%) (Fig. 4). Family medicine scored the highest for the

\begin{tabular}{|c|c|c|c|c|c|}
\hline Demographic data & Family medicine & Internal medicine & Paediatrics & Consulting, all & Surgical, all \\
\hline Median age, years & 54.0 & 36.5 & 40.5 & 42.0 & 35.0 \\
\hline Range, years & $32-65$ & $29-64$ & $30-63$ & $29-65$ & $28-78$ \\
\hline Median duration of practice, years & 14.5 & 8.0 & 9.5 & 10.0 & 4.0 \\
\hline Range, years & $4-32$ & $2-30$ & $1-30$ & $1-32$ & $1-50$ \\
\hline \multicolumn{6}{|l|}{ Gender* } \\
\hline Male, $n(\%)$ & $9(69.2)$ & $12(50.0)$ & $9(56.3)$ & $30(56.6)$ & $49(75.4)$ \\
\hline Female, $n(\%)$ & $4(30.8)$ & $12(50.0)$ & $7(43.7)$ & $23(43.4)$ & $16(24.6)$ \\
\hline Ratio & $2.25: 1$ & $1: 1$ & $1.3: 1$ & $1.3: 1$ & $3.1: 1$ \\
\hline
\end{tabular}

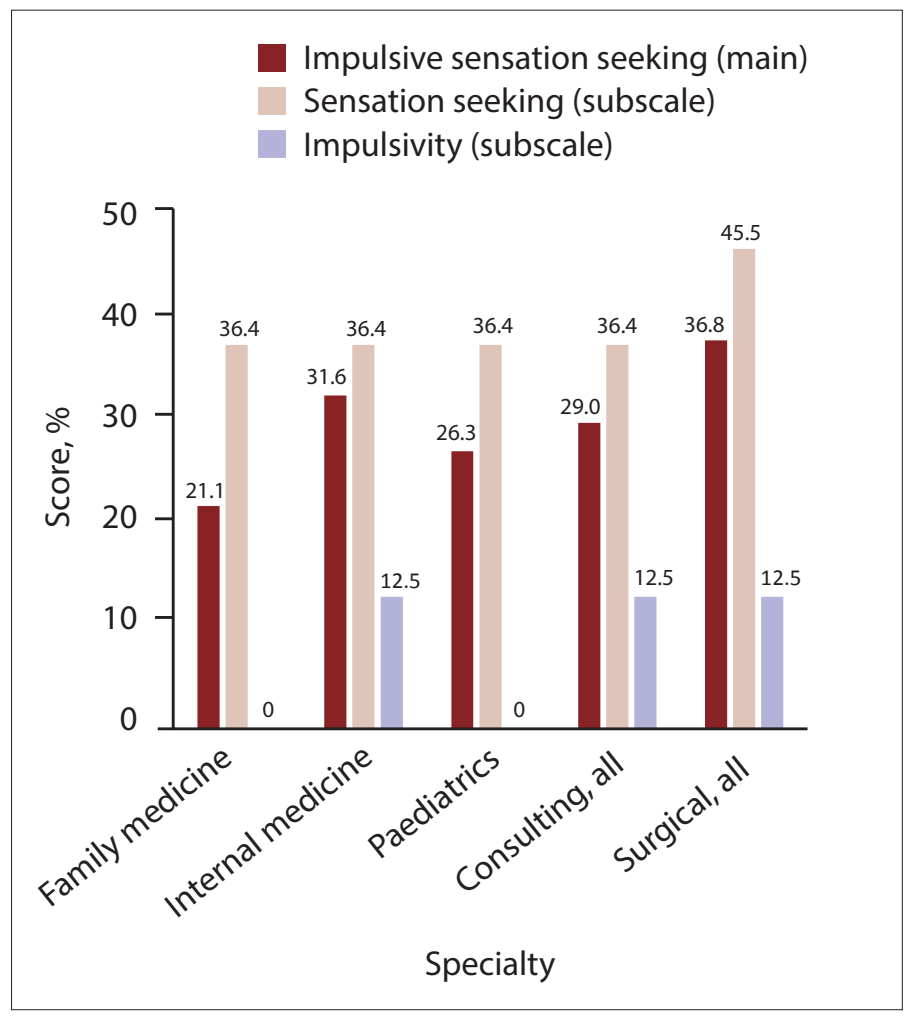

Fig. 1. Median scores of the different specialties of the consulting group, as well as the overall consulting group and the overall surgical group for the factor impulsive sensation seeking.

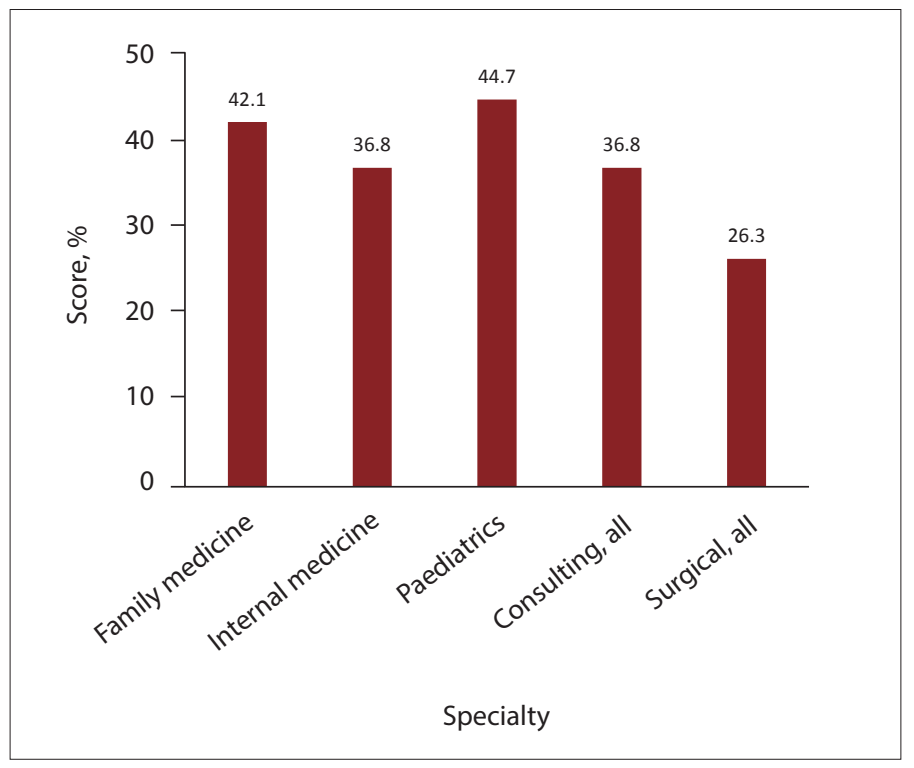

Fig. 2. Median scores of the different specialties of the consulting group, as well as the overall consulting group and the overall surgical group for the factor neuroticism-anxiety.

subscale parties and friends (33.3\%). The consulting group scored lower than the surgical group for sociability $(p=0.01 ; 95 \% \mathrm{CI}-11.8 \% ; 0)$.

\section{Activity}

Internal medicine scored the lowest on the scale for activity (47.1\%), as well as for the subscale general activity (33.3\%) (Fig. 5). Paediatrics had 


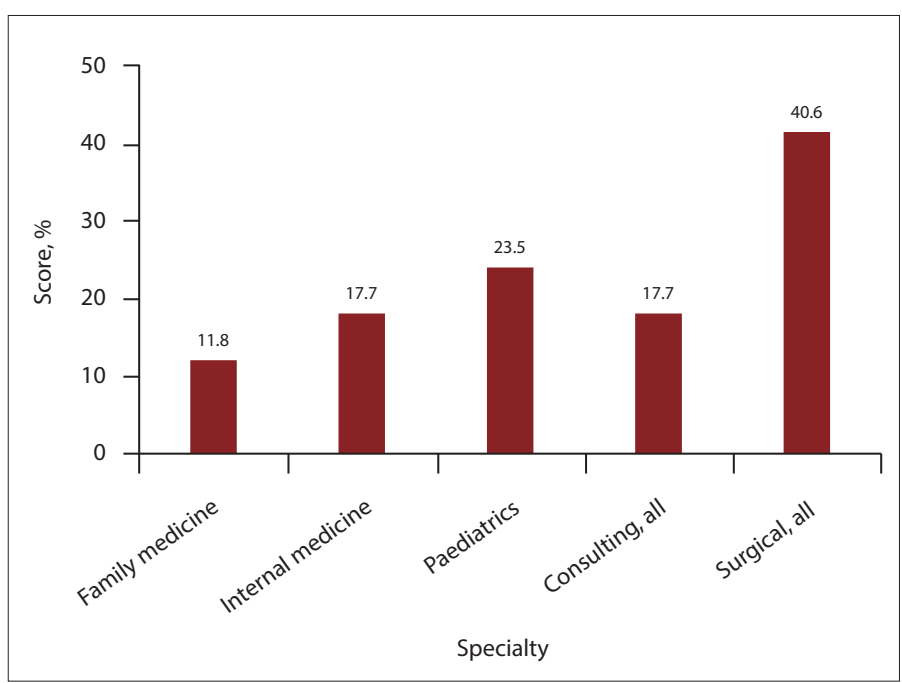

Fig 3. Median scores of the different specialties of the consulting group, as well as the overall consulting group and the overall surgical group for the factor aggression-hostility.

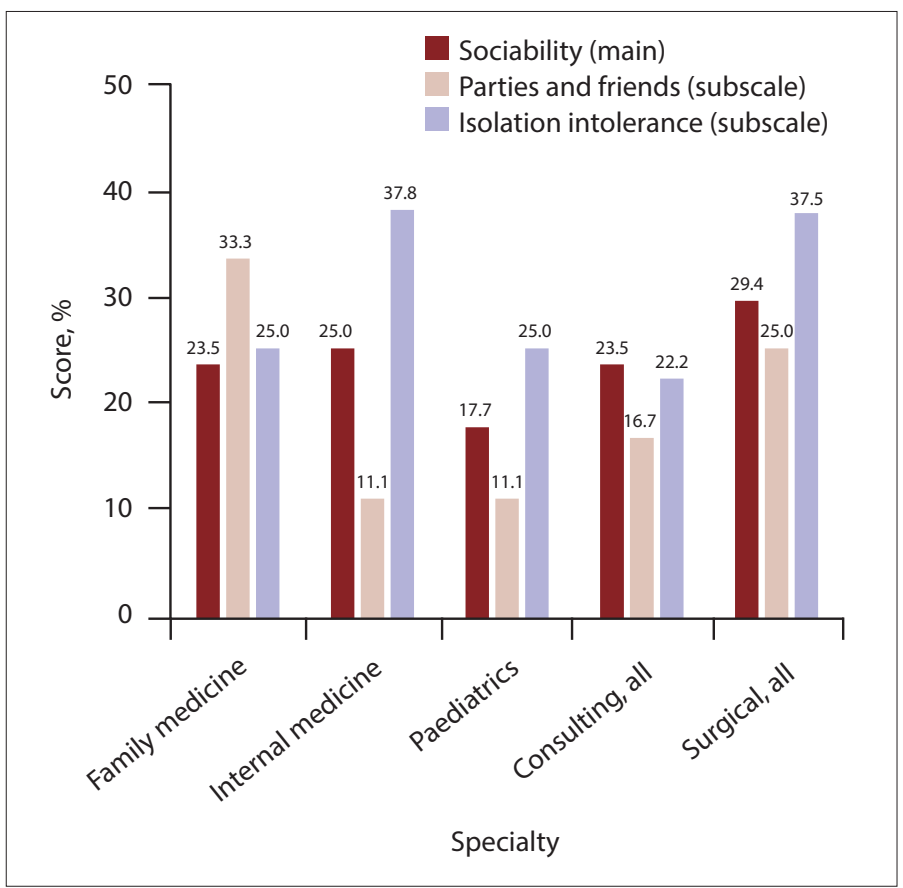

Fig. 4. Median scores of the different specialties of the consulting group, as well as the overall consulting group and the overall surgical group for the factor sociability.

the highest score for the subscale work effort (68.8\%). The consulting group scored significantly lower than the surgical group for activity $(p<0.01$; $95 \%$ CI $-17.6 \% ;-5.9 \%)$, as well as for the subscales general activity $(p=0.06)$ and work effort $(p=0.01)$.

\section{Discussion}

This study was conducted among specialist doctors working at an academic hospital in Bloemfontein. They are therefore either in an academic position or pursuing academic studies. To evaluate a more homogenous group, it was decided that only doctors in the academic setting would be included. Doctors drawn to academics may have a different personality type with different aims in life, compared with doctors in private practice.

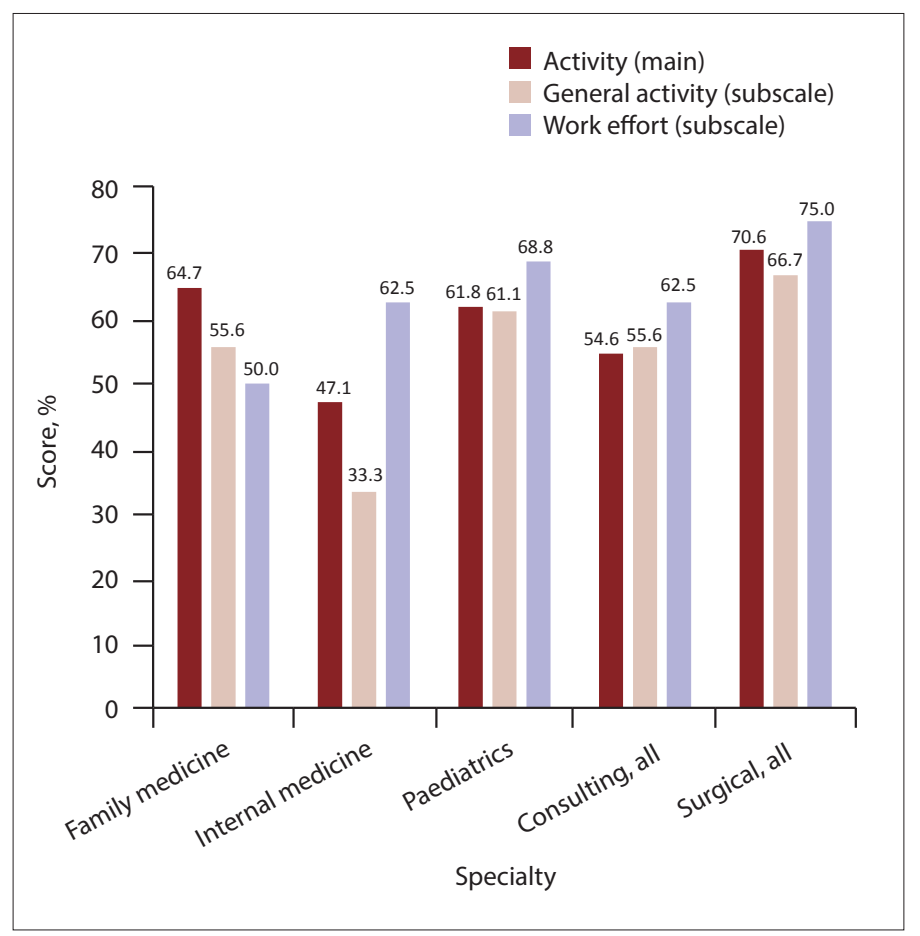

Fig. 5. Median scores of the different specialties of the consulting group, as well as the overall consulting group and the overall surgical group for the factor activity.

\section{Family medicine}

Family medicine scored the lowest for the factor aggression-hostility. A possible explanation could be the higher median age of this group, causing aggression and hostility to decrease with increasing maturity and experience, while the patient-orientated nature of the practice necessitates non-aggressive behaviour.

Family medicine also had the lowest impulsive sensation-seeking scores. The results suggest that family medicine specialists are in general not risktaking or novelty-seeking individuals. This may again be owing to the higher median age, or the broader scope of the specialty, making it less exciting to the more adrenaline-driven individuals. However, one would expect that a specialty with a broad scope would allow for greater diversity, which is an archetypical need for impulsive and sensation-seeking individuals. Impulsivity was very low across all the specialties. This may be because of high expectations of professional conduct and intolerance to impulsivity in the work environment of medical specialists.

Sociability is 'the tendency to interact with others, enjoyment in being with others, and apparent intolerance for social isolation. Although sociability may not be a crucial aspect of an effective doctor's personality, proficiency in interpersonal and communication skills is highly regarded by patients, according to a study on patient perspectives on physician behaviour. ${ }^{[13]}$ For the sociability scale, family medicine had the lowest score for isolation intolerance (25.0\%) and the highest for parties and friends (33.3\%). The reverse is true for the other two specialties. It appears, therefore, that family medicine specialists may not so much be asocial as being highly tolerant of social isolation.

An example of a statement from the ZKPQ for work effort is: 'I like a challenging task much more than a routine one', and for general activity: 'I like to be doing things all of the time.' Although family medicine scored lowest of all specialties in the subscale work effort (50.0\%), it scored highest 
in the activity (64.7\%) scale. This may indicate that these specialists prefer being busy with multiple activities than focusing on a single challenging task.

\section{Internal medicine}

Internal medicine recorded scores with the widest difference between two subscales: work effort (62.5\%) and general activity (33.3\%). This could reflect a penchant for hard and challenging work, but not a busy lifestyle. Internal medicine also had the lowest score of the consulting specialties for neuroticism-anxiety and the highest score for sociability, although only marginally in both scales. Under isolation intolerance, internal medicine had a higher score than the overall score for consulting and surgical groups.

\section{Paediatrics}

The highest score for neuroticism-anxiety was observed in this specialty. The researchers hypothesise that paediatricians' preference for caring for and interacting with children may correspond with greater sensitivity and anxiety. In other words, the specialty draws more sensitive and concerned individuals who tend to be more anxious themselves. Hojat and Zuckerman ${ }^{[3]}$ showed that there was a greater need in childcare for "harm avoidance', a trait that they found to correlate with high neuroticism and anxiety.

Paediatrics scored the lowest in sociability. This was surprising, as the authors believed that interaction with children would be associated with a sociable disposition. An aversion to social pressure may be associated with high neuroticism-anxiety, low sociability and a preference for interacting with children and mothers. Furthermore, social interaction with children is different from interacting with peers. It may be that the lack of 'typical' socialising with patients causes a decrease in sociability over time.

Even though paediatrics had low aggression-hostility scores (23.5\%), these were still higher than those of the other two consulting specialties. This was surprising, as it was assumed that people who interact with children would be the least aggressive.

\section{Consulting group v. surgical group}

The surgical group was found to have higher impulsive sensation-seeking scores than the consulting group. This may be attributed to the physically stimulating nature of surgical procedures that attracts 'novelty-seeking' individuals.

The consulting group had higher neuroticism-anxiety, which may be due to differences in the doctor-patient relationship. The consulting group was much less aggressive. This may also be attributed to a difference in patient interaction, with consulting specialists needing to be approachable, as their work is centred on consultations, not procedures.

The consulting group had a lower sociability score than the surgical group. One explanation for this is the higher neuroticism-anxiety of this group, which implies more sensitivity to criticism. Moreover, the need for teamwork during surgical procedures, greater academic emphasis of consulting specialties, higher median age of consulting specialists (42 v. 35 years), and difference in role models may have played a part. The ZKPQ study ${ }^{[3]}$ among medical students found that sociability correlated positively with aggression-hostility. However, it was expected that these two factors would be inversely related to each other. Rudeness, impatient behaviour and a quick temper would not normally be associated with good interpersonal relationships. It may be that a limited amount of aggression-hostility could be perceived as social 'dominance', and therefore greater success in socialising with others. The ZKPQ study ${ }^{[3]}$ showed no statistically significant difference in sociability between the medical student groups tested.

A study of 590 medical students in Saudi Arabia, ${ }^{[14]}$ using the ZKPQ, found that those interested in surgical specialties scored higher than all other specialty choice groups on all factors, including neuroticism-anxiety, except for activity.

\section{Study limitations}

The small population and sample sizes for each specialty made generalisation and comparison difficult, and prevented more in-depth analysis into the influence of gender, age and years in field of specialty on personality factors. The study only comprised doctors at an academic hospital, and may not fully reflect the personality types of doctors working in the greater private and public sectors.

Sampling bias was also of concern, as the completion of the questionnaires depended on the availability of doctors. Gender and age were measured, but due to limited sample size, it was not possible to correct for these biases. Recruiting of participants at meetings may have systematically excluded doctors with specific personalities, who do not attend meetings.

The ZKPQ does not exhaust the entire range of personality characteristics that any person can possess, and it is emphasised that the results merely provide indications and suggestions and should not be used to place any individuals into 'boxes'. However, all the specialties had a great range in the minimum and maximum values, demonstrating that specialties are not exclusive of any personality types, but that there may be specialties that are more suited to a person's personality than others.

Although published research generally agrees regarding the grouping of family medicine, internal medicine and paediatrics specialties as primary care/people-orientated/consulting, comparison with other studies are hampered by some differences in categorisation of specialties, e.g. Mehmood et al. ${ }^{[14]}$ grouped anaesthesiology with hospital-based procedures, whereas we grouped it with surgical specialties.

\section{Conclusion}

The study helped to characterise the personalities of doctors in some specialties, as demonstrated in Bloemfontein, and revealed some of the differences between findings regarding local specialists and results from studies elsewhere.

\section{Recommendations}

Potential future research efforts that we recommend include: $(i)$ a comparison of personality factors, such as sociability for different professions; (ii) a study comparing the personalities of consulting specialists with correction for gender; and (iii) a study on the level of neuroticism-anxiety of South Africans in general.

It could not be confidently ascertained whether choice of specialty was a product of personality, or whether personality was a product of specialty. A study on whether conforming to the personality norm of one's profession has an impact on career satisfaction will substantiate research on personalities and professions. It is known that certain personality traits impact on career satisfaction, ${ }^{[15-17]}$ but it is not known whether conforming to the norm is related to career satisfaction. 
Acknowledgements. The researchers would like to acknowledge the following people for their assistance, guidance and suggestions: Dr Chris Snowdowne, the initial study leader, who formulated the idea for the study and guided the researchers throughout the planning phase; Prof. Marvin Zuckerman, from the University of Delaware, USA, for generously providing the ZKPQ free of charge for use in the study; Mr Jacques le Roux, a colleague of the researchers, for suggesting the recommendation of investigating whether matching the personality norm of one's field is associated with career satisfaction; the Research Committee of the School of Medicine, UFS, for providing funding for printing; and Ms T Mulder, medical editor, School of Medicine, UFS, for technical and editorial preparation of the manuscript.

Author contributions. RvA, AR, GC, CS and JTK developed the protocol, designed the questionnaire, performed the data collection and coding of questionnaires and wrote a draft report as part of an undergraduate research module. WJS was the supervisor of this study, contributing to the planning, interpretation of results and writing of the manuscript. GJ assisted with the planning of the project, performed the analysis of the data, and assisted with the interpretation of the data and the writing of the manuscript.

\section{Funding. None.}

Conflicts of interest. None.
3. Hojat M, Zuckerman M. Personality and specialty interest in medical students. Med Teach 2008;30(4):400-406. https://doi.org/10.1080/01421590802043835

4. Zuckerman M. Zuckerman-Kuhlman Personality Questionnaire. In: De Raad B, Perugini M, eds. Big Five Assessment (ZKPQ), and Alternative Five-Factorial Model. Seattle: Hogrefe \& Huber, 2002:377-396.

5. Vaidya NA, Sierles FS, Raida MD, Fakhoury FJ, Przybeck TR, Cloninger CR. Relationship between specialty choice and medical student temperament and character assessed with Cloninger inventory. Teach Learn Med 2004;16(2):150-156. https://doi.org/10.1207/s15328015tlm1602_6

6. Rathi AA, Prabhugaonkar SV, Jadhav BS, Shanker S, Dhavale HE. Study of personality factors in postgraduate medical students. Bombay Hosp J 2008;50(2):245-249.

7. Freeman B. The Ultimate Guide to Choosing a Medical Specialty. 3rd ed. New York: McGraw-Hill Medical, 2012. 8. Coombs RH, Fawzy FI, Daniels ML. Surgeons' specialities: The influence of medical school. Med Educ 1993;27(4):337-343.

9. Zimet CN, Held ML. The development of views of specialties during four years of medical school. J Med Educ 1975;50(2):156-166

10. Linn BS, Zeppa R. Does surgery attract students who are more resistant to stress? Ann Surg 1984;200(5):638-643. https://doi.org/10.1097/00000658-198411000-00014

11. Zuckerman M, Kuhlman MD, Thornquist M, Kiers H. Five (or three) robust questionnaire scale factors of Zuckerman M, Kuhlman MD, Thornquist M, Kiers H. Five (or three) robust questionnaire scale factors of
personality without culture. Personal Individ Diff 1991;12(9):929-941. https://doi.org/10.1016/0191-8869(91)90182-

12. Gomà-i-Freixanet M, Wismeijer AA, Valero S. Consensual validity parameters of the Zuckerman-Kuhlman personality questionnaire: Evidence from self-reports and spouse reports. J Personal Assess 2005;84(3):279-286. https://doi.org/10.1207/s15327752jpa8403_07

3. Bendapudi NM, Berry LL, Frey KA, Parish JT, Rayburn WL. Patients' perspectives on ideal physician behavior Mayo Clin Proc 2006;81(3):338-344. https://doi.org/10.4065/81.3.338

4. Mehmood SI, Khan MA, Walsh KM, Borleffs JC. Personality types and specialist choices in medical students. Med Teach 2012;35(1):63-68. https://doi.org/10.3109/0142159X.2012.731104

15. Lounsbury JW, Loveland JM, Sundstrom ED, Gibson LW, Drost AW, Hamrick FL. An investigation of personality traits in relation to career satisfaction J Career Assess 2003:11(3):287-307. https://doi.org/10.1177/ 1069072703254501

16. Levy JJ, Richardson JD, Lounsbury JW, Stewart D, Gibson LW, Drost AW. Personality traits and career satisfaction of accounting professionals. Individ Diff Res 2011;9(4):238-249.

17. Judge TA, Heller D, Mount MK. Five-factor model of personality and job satisfaction: A meta-analysis. J Appl Psychol 2002;87(3):530-541. https://doi.org/10.1037/0021-9010.87.3.530

Accepted 9 November 2017 